\title{
Generic $R$-transform for Invariant Pattern Representation
}

\author{
Thai V. Hoang ${ }^{1,2}$, and Salvatore Tabbone ${ }^{1}$ \\ ${ }^{1}$ LORIA, UMR 7503, Université Nancy 2, 54506 Vandœuvre-lès-Nancy, France \\ ${ }^{2}$ MICA, UMI 2954, Hanoi University of Science and Technology, Hanoi, Vietnam
}

\begin{abstract}
The beneficial properties of the Radon transform make it an useful intermediate representation for the extraction of invariant features from pattern images for the purpose of indexing/matching. This paper revisits the problem with a generic view on a popular Radon-based pattern descriptor, the $R$-signature, bringing in a class of descriptors spatially describing patterns at all the directions and at different levels. The domain of this class and the selection of its representative are also discussed. Theoretical arguments validate the robustness of the generic $R$-signature to additive noise and experimental results show its effectiveness.
\end{abstract}

\section{Introduction}

Many descriptors have been proposed in literature for the extraction of pattern's invariant features $[19,21]$ using techniques that allow invariance to rotation, scaling, translation (RST) or their combinations. Translation and scaling invariance could be obtained by using the Fourier and the Mellin transforms respectively [12]; rotation invariance by performing the Fourier transform on the circular coordinate of the pattern image in polar space [20], etc. However, the task of combining several techniques to have all RST while guaranteeing the discrimination power of the invariant features is challenging and has attracted attention of many researchers. Most of the existing methods do not allow invariance to all RST, they usually require normalizations for the unavailability of any of RST. For example, methods based on the theory of moments [16] usually normalize input pattern images regarding their size and centroid position, which is required to coincide with the origin of the coordinate system. These normalizations usually introduce errors, are sensitive to noise, and thus induce inaccuracy in the later matching process.

Radon-based methods are different from the others in the sense that Radon transform is used as an intermediate representation upon which invariant features are extracted from. Let $f(x, y) \in \mathbb{R}^{2}$ be a $2 \mathrm{D}$ function, $L(\theta, \rho)$ be a straight line in $\mathbb{R}^{2}$ represented using a parameter $t$ by:

$$
(x(t), y(t))=t(\sin \theta,-\cos \theta)+\rho(\cos \theta, \sin \theta),
$$

where $\theta$ is the angle $L$ makes with the $y$ axis and $\rho$ is the distance from the origin to $L$. The Radon transform [3] of $f$, denoted by $\mathcal{R}_{f}$, is a function defined on the space of lines $L$ by the line integral along each line:

$$
\mathcal{R}_{f}(L)=\mathcal{R}_{f}(\theta, \rho)=\int_{-\infty}^{\infty} f(x(t), y(t)) d t .
$$

The following beneficial properties of the Radon transform can be easily obtained from this definition:

- $P 1$ : The Radon transform of $f(x, y)$ is periodic in the variable $\theta$ with period $2 \pi$ and semi-symmetric with $\mathcal{R}_{f}(\theta, \rho)=\mathcal{R}_{f}(\theta \pm \pi,-\rho)$.

- $P 2$ : A translation of $f(x, y)$ by a vector $\vec{u}=\left(x_{0}, y_{0}\right)$ results in a shift of $\mathcal{R}_{f}(\theta, \rho)$ in the variable $\rho$ by a distance $d=x_{0} \cos \theta+y_{0} \sin \theta$ equal to the projection of $\vec{u}$ on the line $x \cos \theta+y \sin \theta=\rho$.

- P3: A rotation of $f(x, y)$ by an angle $\theta_{0}$ implies a shift of $\mathcal{R}_{f}(\theta, \rho)$ in the variable $\theta$ by a distance $\theta_{0}$.

- P4: A scaling of $f(x, y)$ by a factor $\alpha$ results in a scaling of the $\rho$ coordinate and the amplitude of $\mathcal{R}_{f}(\theta, \rho)$ by a factor $\alpha$ and $\frac{1}{\alpha}$ of the transform data respectively.

Thus, the Radon transform converts RST transformations applied on a pattern image into transformations in the radial (for translation and scaling) and angular (for rotation) coordinates of the Radon image [5]. Current techniques usually exploit this encoded information to define invariant descriptors. Fig. 1 illustrates the effect of RST transformations on the Radon image, the pattern image $I_{2}$ is a scaled, rotated, and translated version of the image pattern $I_{1}$.

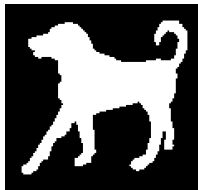

(a) Image $I_{1}$

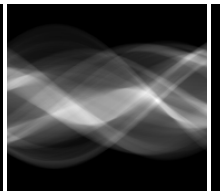

(b) $\mathcal{R}_{I_{1}}(\theta, \rho)$

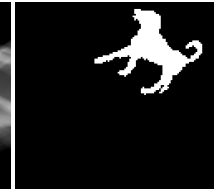

(c) Image $I_{2}$

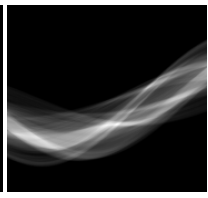

(d) $\mathcal{R}_{I_{2}}(\theta, \rho)$
Figure 1. The effect of RST transformations on $\mathcal{R}_{I_{k}}(\theta, \rho)$.

\subsection{Previous works}

A pioneer work in this direction is the $R$-transform, which gives rise to the $R$-signature [15], obtained by using an integral function and then Fourier transform for the radial and 
angular coordinates of the Radon image respectively. Similarly, the $\Phi$-signature [10] is computed by using an integral function along the angular coordinate of the Radon image to get rotation invariance. Invariance to translation and scaling is made possible by normalization. The strength of these two approaches is simplicity, however, the obtained signatures have low discriminatory power as there is a loss of information in the compression process from the Radon image to 1D signatures. Moreover, the required normalization for the $\Phi$-signature prevents it from being applied to noisy images.

There was an effort to apply the 2D Fourier-Mellin transform on the Radon image [17] to get invariance to rotation and scaling. In this approach, Mellin and Fourier transforms are applied on the radial and angular coordinates of the Radon image respectively. The main weakness of this approach is the lack of translation invariance. This drawback has been overcome in [6] by replacing the Mellin transform by the 1D Fourier-Mellin transform for the radial coordinate. Recently, a set of spectral and structural features has also been extracted from the Radon image for pattern description [2]. In this set, the "degree of uniformity" is essentially the $R$-signature and the "longest line" is the information encoded in the generic $R$-signature described in this paper. However, and more importantly, this set of features is not invariant to rotation and, consequently, in the matching step, these features need to be rotated to all possible angles corresponding to potential pattern's orientations in order to compute patterns' similarity. Long matching time may prevent the application of this approach in real systems.

Among the Radon-based pattern descriptors, $R$-signature is the most popular due to its simplicity and has been successfully applied to several applications, e.g. symbol recognition [13], activity recognition $[14,18]$, and orientation estimation [7]. This paper provides a generic view on the $R$-transform and the $R$-signature while maintaining their beneficial properties, leading to two main theoretical contributions. The first is a better understanding of the discrimination power of the generic $R$-signature, which results from the exploitation of variation in the accumulation of the pattern image along all the parallel lines, leading to an increase in performance. The second is the ability of the generic $R$-transform to represent dominant directions of patterns, even in the present of noise, resulting in the superiority of the generic $R$-signature on noisy datasets over comparing methods.

The conventional $R$-signature is briefly reviewed in the following subsection. Section 2 presents the generic $R$ signature along with its geometric interpretation and its properties. A discussion on the meaningful domain of the generic signature is carried out in Section 3. Theoretical arguments on its robustness to additive noise and its ability to encode dominant directions of patterns are presented in Section 4. Experimental results are given in Section 5 and finally conclusions are drawn in Section 6.

\subsection{The $R$-signature}

The $R$-transform of a 2D function $f(x, y) \in \mathbb{R}^{2}$ proposed in [15] has the following definition:

$$
R_{f 2}(\theta)=\int_{-\infty}^{\infty} \mathcal{R}_{f}^{2}(\theta, \rho) d \rho .
$$

The integration computed along the radial axis of the Radon image in Eq. (3) makes $R_{f 2}(\theta)$ invariant to translation and scaling, except for a multiplicative factor $\frac{1}{\alpha^{3}}$ resulting from the scaling factor $\alpha$ in $f(x, y)$, and periodic with period $\pi$. Furthermore, in order to have a representation totally invariant to RST transformations, the authors in [15] has proposed to use the magnitude of the Fourier transform performed on $R_{f 2}(\theta)$ normalized by the DC component as:

$$
F R_{f 2}(\xi)=\left|\frac{\int_{0}^{\pi} R_{f 2}(\theta) e^{-2 \pi i \theta \xi} d \theta}{\int_{0}^{\pi} R_{f 2}(\theta) d \theta}\right|
$$

and the $R$-signature of $f(x, y)$ is originally defined as $\left[F R_{f 2}(1), F R_{f 2}(2), \ldots, F R_{f 2}(\pi)\right]$. It is thus not difficult to prove that $F R_{f 2}(\xi)$ is invariant to RST transformations.

\section{Generalization of the $R$-signature}

The $R$-signature defined above has been extended in [15] for shape images by computing $F R_{f_{i}, 2}(\theta)$, where $f_{i}$ $(i=0 \rightarrow 7)$ are derived from $f$ by segmenting its distance transform at 8 equidistant levels. This extension leads to an increase in the discrimination power of the $R$-signature because the derived shape images $f_{i}$ preserve the topology of $f$ and, when $i$ increases, the level of deformation decreases. This extension, however, is inapplicable to grayscale images.

Another extension is proposed in this section by generalizing the $R$-transform in Eq. (3) to further increase the discrimination power. The $R$-transform in Eq. (3) is just a special case of a class of transforms sharing beneficial properties for pattern representation and matching. This section provides their definition and their geometric interpretation in the spatial space along with the properties of the generic $R$-signature defined based on these transforms.

\subsection{Definition}

For a 2D function $f(x, y) \in \mathbb{R}^{2}$ and $m \in \mathbb{R}$, the generic $R$-transform of $f(x, y)$, denoted as $R_{f m}(\theta)$, is defined as:

$$
R_{f m}(\theta)=\int_{-\infty}^{\infty} \mathcal{R}_{f}^{m}(\theta, \rho) d \rho .
$$

Evidently, by setting $m=2, R_{f m}(\theta)$ becomes $R_{f 2}(\theta)$ in Eq. (3). The utilization of the exponent $m$ as a parameter makes $R_{f m}(\theta)$ a generic version of $R_{f 2}(\theta)$. Furthermore, by varying the value of $m$, a class of transforms is obtained and this in turn results in a class of signatures. The derivation of the generic $R$-signature, $F R_{f m}(\xi)$, from $R_{f m}(\theta)$ follows strictly the process described in Subsection 1.2. 


\subsection{Geometric interpretation}

Recall that the value of $\mathcal{R}_{f}(\theta, \rho)$ is the result of a line integral of $f(x, y)$ along the line $L(\theta, \rho)$. Consequently, the generic $R$-transform defined based on $\mathcal{R}_{f}(\theta, \rho)$ by computing integrals over the variable $\rho$ has some geometric interpretations. The generic $R$-transform of $f, R_{f m}(\theta)$, in Eq. (5) is basically an integral of $\mathcal{R}_{f}^{m}(\theta, \rho)$ computed over the variable $\rho$ of the Radon image. In other words, this integral is computed by using the result of line integral along all the lines parameterized by a fixed value of $\theta$ and different values of $\rho$. Sharing the same value of $\theta$ means that these lines are parallel in the spatial space (Fig. 2) and $R_{f m}(\theta)$ encodes the spatial information of the pattern image $f(x, y)$ in the direction making an angle $\theta$ with the $y$ axis. Encoding $f(x, y)$ at different directions is possible by varying $\theta$ to have $R_{f m}(\cdot)$ and $R_{f m}(\cdot)$ could be then interpreted as containing the encoded spatial information of $f(x, y)$ at all the directions. Furthermore, the role of the exponent $m$ in Eq. (5), besides setting up a class of transforms, is to make $R_{f m}(\cdot)$ discriminatory at different values of $m$ by exploiting the variation in $\mathcal{R}_{f}(\theta, \cdot)$.

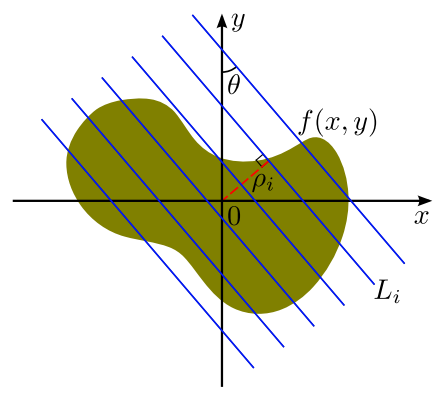

Figure 2. Geometric illustration of the generic $R$-transform.

\subsection{Properties}

From Eq. (5), it is straightforward that the generic $R$ transform of $f(x, y)$ is periodic in the $\theta$ coordinate with period $\pi$ and is invariant to translation. A rotation of $f(x, y)$ by an angle $\theta_{0}$ implies a shift of $R_{f m}(\theta)$ by a distance $\theta_{0}$. A scaling of $f(x, y)$ by a factor $\alpha$ results in a scaling of the amplitude of $R_{f m}(\theta)$ by a factor $\frac{1}{\alpha^{m+1}}$. Thus, the generic $R$-signature defined based on the generic $R$-transform as in Subsection 1.2 is totally invariant to RST transformations. Fig. 3 illustrates the properties of the generic $R$-transform by using the two pattern images $I_{1}$ and $I_{2}$ in Fig. 1 . The value of $R_{I_{k} m}(\cdot)$ has been normalized by the area they make with the $\theta$ axis, $\int_{0}^{\pi} R_{I_{k} m}(\theta) d \theta$, for better viewing.

\section{The domain for the exponent $m$}

The generic $R$-transform, as defined in Eq. (5), theoretically produces a class of transforms having an infinite number of members obtained by varying the value of the exponent $m$. However, in reality, the domain for reasonable

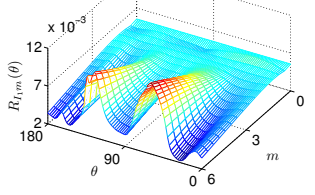

(a) $R_{I_{1} m}(\theta)$

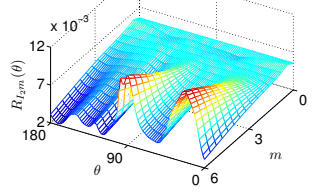

(b) $R_{I_{2} m}(\theta)$
Figure 3. Illustration of the properties of the generic $R$-transform.

values of $m$ is limited, not all the space $\mathbb{R}$. First, the generic $R$-transform has one singularity at $m=1$ :

$$
R_{f 1}(\theta)=\int_{-\infty}^{\infty} \mathcal{R}_{f}^{1}(\theta, \rho) d \rho=\sum_{x, y=-\infty}^{\infty} f(x, y) .
$$

Thus, $R_{f 1}(\cdot)$ contains no discriminatory information, except for scaling. Moreover, when $m$ reaches $\infty$ :

$$
\lim _{m \rightarrow+\infty} R_{f m}(\theta)=\lim _{m \rightarrow+\infty} \int_{-\infty}^{\infty} \mathcal{R}_{f}^{m}(\theta, \rho) d \rho=+\infty,
$$

implying that in practice $m$ cannot have excessive big values. In addition, negative value of $m$ should also be avoided due to the sensitivity of negative power function to very small values, introducing artifacts to the signature. Indeed, at the furthest point from the pattern centroid's position, the intersection between the tangent line $L\left(\theta^{\star}, \rho^{\star}\right)$ and the pattern image $f(x, y)$ has infinitesimal length, inducing a very small value at $\mathcal{R}_{f}\left(\theta^{\star}, \rho^{\star}\right)$. Taking the negative power of this value produces a very big value.

By definition, the Radon transform is essentially the projection of the spatial pattern image $f(x, y)$ along all the lines $L(\theta, \rho)$, making it robust to additive noise [8]. However, as the generic $R$-transform is defined based on the exponentiation of $\mathcal{R}_{f}(\theta, \rho)$, the remaining variation due to noise in $\mathcal{R}_{f}(\theta, \rho)$ will result in variation in $R_{f m}(\cdot)$ at different levels according to the value of the exponent $m$. Too high value of $m$ will cause high variation in $R_{f m}(\cdot)$ and make it very different from the ideal analytical values. The heavily deformed $R_{f m}(\cdot)$ due to noise will make the representation inappropriate for recognition/matching.

Sampling and quantization could be considered as processes that add noise to the original analytical pattern image. In this sense, the image processed by digital computers is a noisy image and the variation in $\mathcal{R}_{f}(\theta, \rho)$ is unavoidable. Fig. 4 illustrates the sensitivity of the generic $R$-transform to sampling and quantization. The image $I_{2}$ in Fig. 4(b) is a sampled and quantized version of an analytical triangle $I_{1}$ in Fig. 4(a) and has a size of $100 \times 100$ pixels. The value of $R_{I_{k} m}(\cdot)$ with $k=1,2$ has been normalized by the area they make with the $\theta$ axis, $\int_{0}^{\pi} R_{I_{k} m}(\theta) d \theta$, for better viewing. The difference in the normalized value of the generic $R$-transform of $I_{1}$ and $I_{2},\left|R_{I_{2} m}(\theta)-R_{I_{1} m}(\theta)\right|$ shows that a higher value of $m$ will result in bigger difference, meaning a more severe deformation in $R_{I_{2} m}(\theta)$. 


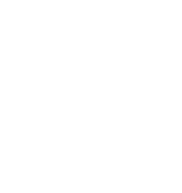

(a) Image $I_{1}$

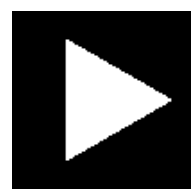

(b) Image $I_{2}$

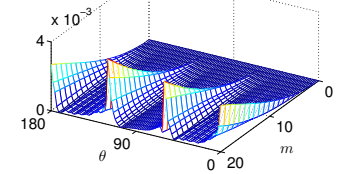

(c) $\left|R_{I_{2} m}(\theta)-R_{I_{1} m}(\theta)\right|$
Figure 4 . The sensitivity to sampling and quantization.

\section{Robustness to noise}

Radon transform has been proved to be robust to additive noise and, as a result, pattern descriptors defined based on the Radon transform are usually more robust to additive noise than the others. This section discusses the robustness to noise of the generic $R$-signature and its ability in representing dominant directions of patterns. The robustness to noise of the generic $R$-signature has its roots not only from the noise-suppressing property of the Radon transform but also from its ability of encoding pattern's dominant directions. Indeed, due to the exponentiation insides the integral in Eq. (5), the contribution of $\Psi(\theta)=\mathcal{R}_{f}\left(\theta, \rho^{\star}\right)$ with $\rho^{\star}=$ $\operatorname{argmax}_{\rho} \mathcal{R}_{f}(\theta, \rho)$ to $R_{f m}(\theta)$ increases as $m$ increases, and:

$$
\lim _{m \rightarrow+\infty} \frac{\Psi^{m}(\theta)}{R_{f m}(\theta)}=1
$$

This means that, at a reasonable high value of $m, R_{f m}(\theta)$ represents the longest intersection of $f(x, y)$ with $L(\theta, \cdot)$ which is similar to the "longest line" feature proposed in [2]. The longest intersection profile $\Psi(\theta)$ of the image $I_{1}$ (normalized by $\max _{\theta} \Psi(\theta)$ for better viewing) in Fig. 1(a) is plotted in Fig. 5(a).

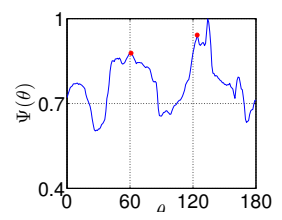

(a) $\Psi(\theta)$

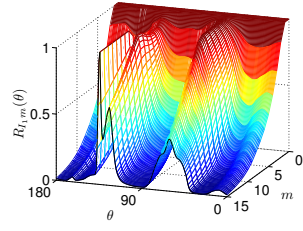

(b) $R_{I_{1} m}(\theta)$

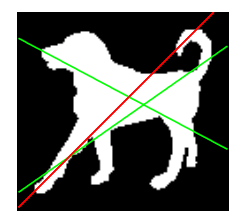

(c) Directions
Figure 5 . The ability to encode pattern's dominant directions.

Similarly, as $m$ increases, the ratio $\frac{R_{f m}(\theta)}{R_{f m}\left(\theta^{\star}\right)}$ with $\theta \neq \theta^{\star}$ and $\theta^{\star}=\operatorname{argmax}_{\theta} R_{f m}(\theta)$ exponentially decreases, and:

$$
\lim _{m \rightarrow+\infty} \frac{R_{f m}(\theta)}{R_{f m}\left(\theta^{\star}\right)}=\delta_{\theta \theta^{\star}},
$$

where $\delta_{\theta \theta^{\star}}=\left[\theta=\theta^{\star}\right]$ is the Kronecker delta function. This means that when $m$ is reasonably high, $R_{f m}(\theta)$ encodes only the direction $\theta^{\star}$, corresponding to the longest intersection between $f(x, y)$ and all the lines $L(\cdot, \cdot)$.

In real applications, Eqs. (8) and (9) do not hold as $m$ does not have high enough value due to the sensitivity of the generic $R$-transform to quantization and sampling as discussed in Section 3. However, there is an evolution in the profile of $R_{f m}(\theta)$ as $m$ increases, transforming a constant function into the Kronecker delta function. During this process, the information encoded by $R_{f m}(\theta)$ also changes, roughly from all directions to the only direction $\theta^{\star}$. The interpretation here is that the dominant directions of $f(x, y)$ are encoded at different levels, depending on $m$. Illustration of this evolution is depicted in Fig. 5(b). It is observed that the three ridges in the surface of the normalized $R_{f m}(\theta)$ correspond to the three local maxima of $\Psi(\theta)$ in Fig. 5(a), which in turns represent the three dominant directions of $f(x, y)$, as shown in Fig. 5(c). As $m$ increases, the role of these maxima in $R_{f m}(\theta)$ interchanges, however, dominant directions are still reflected in the profile of $R_{f m}(\theta)$.

When the pattern image $f(x, y)$ is contaminated by noise to be $\hat{f}(x, y)$, due to the noise-suppressing property of the Radon transform, the difference in the dominant directions of $f(x, y)$ and $\hat{f}(x, y)$ is statistically small. It can be concluded that $R_{f m}(\theta)$ is robust to additive noise and, as a result, the generic $R$-signature is also robust to additive noise.

\section{Experimental results}

Performance of the generic $R$-signature has been evaluated on two set of experiments on two different sets to demonstrate its robustness to additive 'salt \& pepper' and white noise. The generic $R$-signature has been compared with angular radial transform (ART) [1], generic Fourier descriptor (GFD) [20], Zernike moments [9], R2DFM descriptor [17], and RMF descriptor [6]. Among the selected descriptors for comparison, R2DFM and RFM descriptors are also defined on the Radon transform. These descriptors are selected because they are commonly used and have good reported performance. The measure used for comparison among descriptors is the precision-recall curve from information retrieval.

The robustness of the generic $R$-signature to additive "salt $\&$ pepper' noise has been demonstrated using 6 logo datasets generated from the first 25 images of the UMD Logo dataset [4] (shown in Fig. 6). Each of these 6 logo datasets consists of 275 images of 25 categories, each category contains 11 images generated by randomly scaling, rotating, translating the original corresponding logo image and then adding 'salt \& pepper' noise to it. Let $d$ be the percentage of pixels flipped by the noise, the value of $d$ for each generated dataset is kept constant and $d$ has 6 possible values ranging from 0 to 0.1 with increment of 0.02 corresponding to 6 datasets. The first dataset with $d=0$ is actually a noiseless dataset; its use is intended for checking the RST invariant properties of the generic $R$-signature and comparing descriptors as well as the performance of the generic $R$-signature at high value of $m$. The values of $d$ of the other 5 noisy datasets make up an arithmetic progression with common difference 0.02 . These 


\section{L9图 K - (3)}

Figure 6. 25 logo images from the UMD Logo dataset.

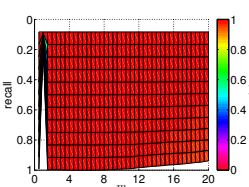

(a) $d=0$

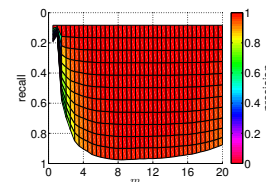

(d) $d=0.06$

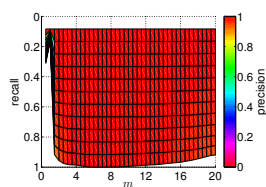

(b) $d=0.02$

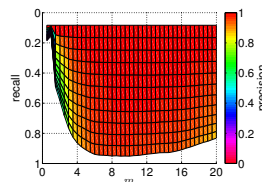

(e) $d=0.08$

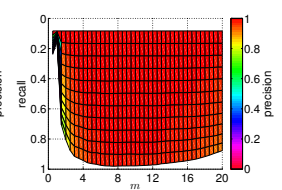

(c) $d=0.04$

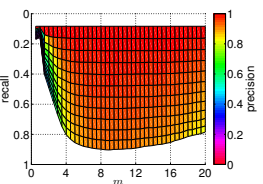

(f) $d=0.1$
Figure 7. Precision-recall curves on the 6 logo datasets.

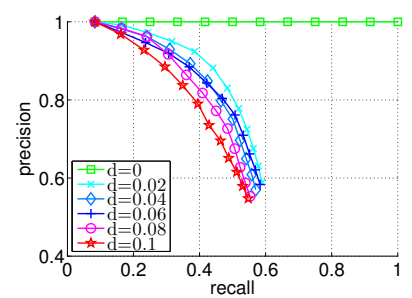

(a) ART moments

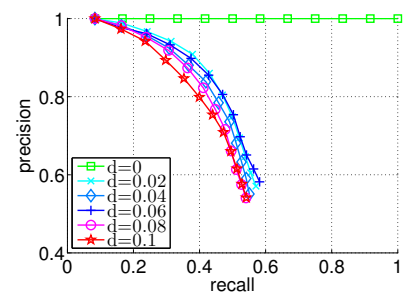

(c) Zernike moments

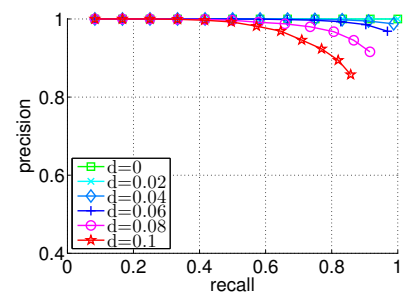

(e) RFM descriptor

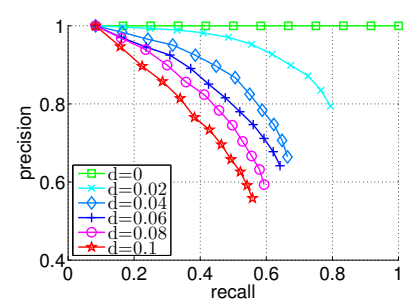

(b) GFD descriptor

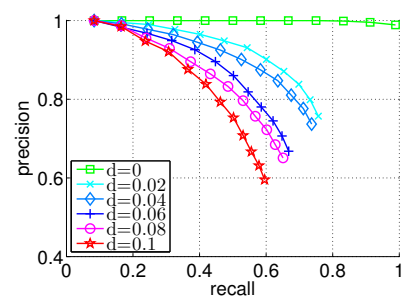

(d) R2DFM descriptor

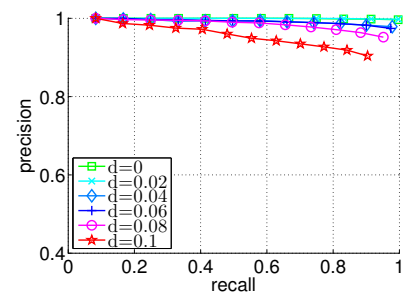

(f) Generic $R$-signature
Figure 8. Performance of comparing descriptors on logo datasets.

5 datasets are, therefore, used to evaluate the robustness of the generic $R$-signature and comparing descriptors at incrementing levels of additive noise.

Fig. 7 provides the precision-recall curves of the generic $R$-signature on the $6 \log$ datasets. In these figures, at a specific value of $m$ in the horizontal axis, there is a precisionrecall curve with recall and precision rates illustrated as the ordinate and the color of the grid points having abscissa $m$. On each dataset, except for the singularity at $m=1$, an increase then a decrease in performance are observed and, in general, the peak in performance is obtained at $m \in[7,10]$. The decrease in performance at high value of $m$ agrees with the discussion on the sensitivity of the generic $R$-signature to sampling/quantization in Section 3. Furthermore, as $d$ increases, the performance of the generic $R$-signature generally deteriorates at each value of $m$. However, the deterioration speed is slow at $m \in[7,10]$, meaning a robustness of the generic $R$-signature to additive noise. This experimental evidence agrees with the theoretical arguments in Section 4.

Comparison results with common descriptors using these 6 datasets are depicted in Fig. 8. In this comparison, the value of $m$ is fixed at 9 . It is observed that the impact of $d$ on the performance of comparing descriptors differs from one to another. As $d$ increases, the curve of all the descriptors generally move downwards and descriptors defined on the Radon transform are more robust to noise than the others. However, among Radon transform-based descriptors, the generic $R$-signature has the highest performance.

Similarly, the robustness of the generic $R$-signature to additive white noise has been demonstrated using 6 object datasets generated from 20 object images from the COIL20 dataset [11] (shown in Fig. 9). Each of these 6 datasets has 220 images of 20 categories, each category contains 11 images. Noisy images are generated from the corresponding noise-free images by randomly scaling, rotating, translating and then adding white noise to them. Let SNR be the signal-to-noise ratio defined as $\mathrm{SNR}=\frac{\sum_{x, y} f^{2}(x, y)}{\sum_{x, y} \eta^{2}(x, y)}$, where $f(x, y)$ is the noise-free image and $\eta(x, y)$ is the added white noise, the value of SNR for each dataset is kept constant and, for each experiment, SNR has 6 possible values $\{0.5,1,2,4,8,16\}$, corresponding to the 6 datasets.

Fig. 10 provides the precision-recall curves of the generic $R$-signature on the 6 object datasets. The evolution of these curves according to $m$ has similar trend with that on the 6 logo datasets in Fig. 7. That is, except for the singularity at $m=1$, an increase then a decrease in performance are observed. Comparison results with common descriptors using these 6 datasets are depicted in Fig. 11. In this comparison, the value of $m$ is fixed at 3. It is observed that ART, GFD, Zernike, and R2DFM descriptors are not robust to additive white noise at all, their performance is similarly poor for different levels of noise. Generic $R$-signature and $\mathcal{R F} \mathcal{M}$ descriptors have comparable and good performance. Their performance is nearly perfect when the noise is weak $(\mathrm{SNR}=16,8,4,2)$.

\section{Conclusions}

This paper has provided a generic view on a popular Radon-based pattern descriptor, the $R$-signature. The generic $R$-signature brings in a class of descriptors having the beneficial properties of the $R$-signature and spatially 


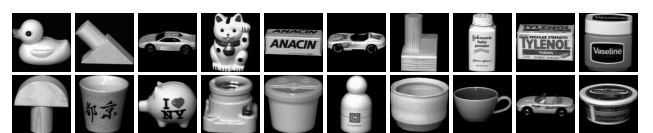

Figure 9. 20 object images from the COIL-20 dataset.

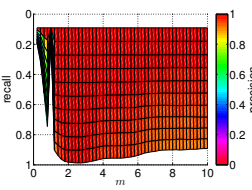

(a) $d=0$

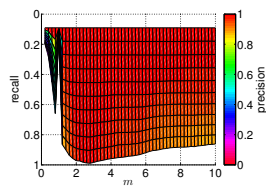

(b) $d=0.02$

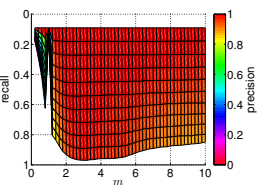

(c) $d=0.04$

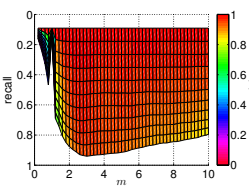

(d) $d=0.06$

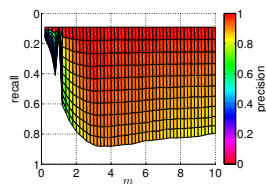

(e) $d=0.08$

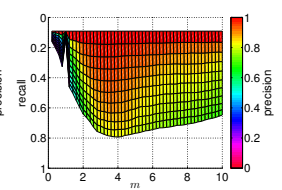

(f) $d=0.1$
Figure 10. Precision-recall curves on the 6 object datasets.

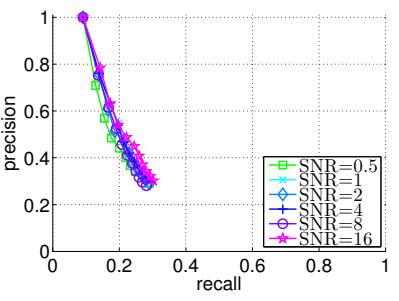

(a) ART moments

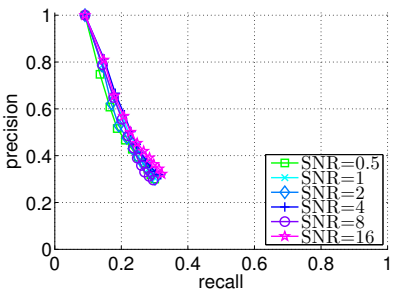

(c) Zernike moments

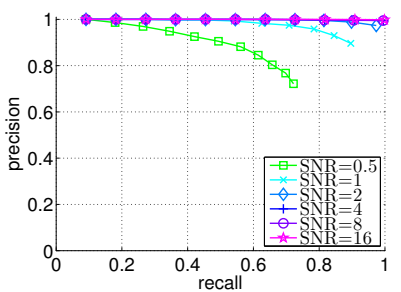

(e) RFM descriptor

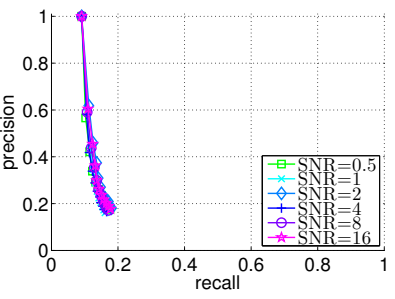

(b) GFD descriptor

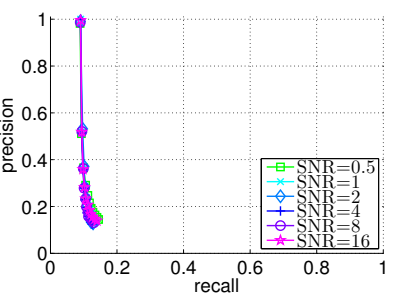

(d) R2DFM descriptor

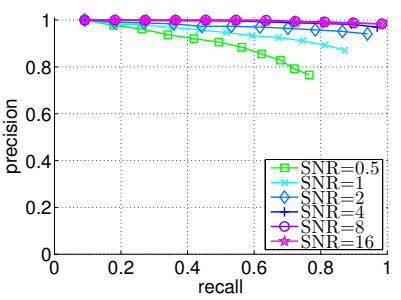

(f) Generic $R$-signature
Figure 11. Performance of comparing descriptors on object datasets.

describing patterns at all the directions and at different levels. Additionally, this generalization gives more flexibility in their definition and, more importantly, the generic $R$ signature has been proved to be robust to additive noise and demonstrated its superiority over existing pattern descriptors on grayscale and binary noisy datasets. Future work investigates optimal combination of generic $R$-signature to have better performance.

\section{References}

[1] M. Bober. MPEG-7 visual shape descriptors. IEEE Trans. Circuits Syst. Video Techn., 11(6):716-719, 2001.

[2] Y. W. Chen and Y. Q. Chen. Invariant description and retrieval of planar shapes using Radon composite features. IEEE Trans. Signal Processing, 56(10-1):4762-4771, 2008.

[3] S. R. Deans. The Radon Transform and Some of Its Applications. Krieger Publishing Company, 1993.

[4] D. S. Doermann, E. Rivlin, and I. Weiss. Applying algebraic and differential invariants for logo recognition. Mach. Vis. Appl., 9(2):73-86, 1996.

[5] H. Hjouj and D. W. Kammler. Identification of reflected, scaled, translated, and rotated objects from their Radon projections. IEEE Trans. Image Processing, 17(3):301-310,2008.

[6] T. V. Hoang and S. Tabbone. A geometric invariant shape descriptor based on the Radon, Fourier, and Mellin transforms. In Proc. ICPR, pages 2085-2088, 2010.

[7] T. V. Hoang and S. Tabbone. Text extraction from graphical document images using sparse representation. In Proc. DAS, pages 143-150, 2010.

[8] K. Jafari-Khouzani and H. Soltanian-Zadeh. Rotationinvariant multiresolution texture analysis using Radon and wavelet transforms. IEEE Trans. Image Processing, 14(6):783-795, 2005.

[9] A. Khotanzad and Y. H. Hong. Invariant image recognition by Zernike moments.IEEE Trans.PAMI,12(5):489-497,1990.

[10] N. Nacereddine, S. Tabbone, D. Ziou, and L. Hamami. Shapebased image retrieval using a new descriptor based on the Radon and wavelet transforms. In Proc. ICPR, 2010.

[11] S. A. Nene, S. K. Nayar, and H. Murase. Columbia object image library (COIL-20). Technical report, Columbia University CUCS-005-96, 1996.

[12] A. D. Poularikas, editor. The Transforms and Applications Handbook. CRC \& IEEE Press, 2000.

[13] O. Ramos-Terrades, E. Valveny, and S. Tabbone. Optimal classifiers fusion in a non-Bayesian probabilistic framework. IEEE Trans. PAMI, 31(9):1630-1644, 2009.

[14] R. Souvenir and K. Parrigan. Viewpoint manifolds for action recognition. EURASIP Journal on Image and Video Processing, 2009:1-13, 2009.

[15] S. Tabbone, L. Wendling, and J.-P. Salmon. A new shape descriptor defined on the Radon transform. Computer Vision and Image Understanding, 102(1):42-51, 2006.

[16] M. R. Teague. Image analysis via the general theory of moments. J. Opt. Soc. Am., 70(8):920-930, 1980.

[17] X. Wang, B. Xiao, J.-F. Ma, and X.-L. Bi. Scaling and rotation invariant analysis approach to object recognition based on Radon and Fourier-Mellin transforms. Pattern Recognition, 40(12):3503-3508, 2007.

[18] Y. Wang, K. Huang, and T. Tan. Human activity recognition based on $R$ transform. In Proc. CVPR, 2007.

[19] J. Wood. Invariant pattern recognition: a review. Pattern Recognition, 29(1):1-17, 1996.

[20] D. Zhang and G. Lu. Shape-based image retrieval using generic Fourier descriptor. Signal Processing: Image Communication, 17(10):825-848, 2002.

[21] D. Zhang and G. Lu. Review of shape representation and description techniques.Pattern Recognition,37(1):1-19,2004. 